\title{
Co-simulation Research Based on Electromagnetic Induction of Wireless Power Transfer
}

\author{
Jiuchao $\mathrm{Li}^{1}$ and Yao Zhao ${ }^{2}$ \\ ${ }^{1,2}$ Engineering Technology Institute of Changchun ,China
}

Keywords: Wireless power transfer; Maxwell; Specification; Co-simulation

\begin{abstract}
It is difficult to realize electromagnetic field and circuit multi domain joint simulation for electromagnetic induction wireless charging system, Using Maxwell and simulation soft ware Joint simulation of standard electromagnetic induction wireless charging system. Maxwell electromagnetic field software is used to publish coil electromagnetic field, inductance and coincidence coefficient, The finite element analysis of ferrite loss is carried out, and the whole circuit is analyzed and verified by using circuit simulation software. The simulation results are compared with the standard, and the accuracy and validity of the simulation results are verified. The joint simulation method has good versatility, and is suitable for the prototype design and verification of the electromagnetic induction wireless charging system, which can effectively shorten the experimental and development cycle, and reduce the R \& D costs.
\end{abstract}

\section{Introduction}

The finite element analysis of ferrite loss is carried out, and the whole circuit is analyzed and verified by using circuit simulation software. The simulation results are compared with the standard, and the accuracy and validity of the simulation results are verified. The joint simulation method has good versatility, and is suitable for the prototype design and verification of the electromagnetic induction wireless charging system, which can effectively shorten the experimental and development cycle, and reduce the $\mathrm{R} \& \mathrm{D}$ costs.

There are 3 kinds of mainstream wireless charging standard: Power There are 3 kinds of mainstream wireless charging standard: Power Wireless Power (A4WP) standard. The Qi standard is the first in the world wireless power consortium, WPC launched "wireless charge" The most popular electromagnetic induction technology Cs7 is adopted at present Maxwell is powerful, accurate and easy to use The 2D / 3D electromagnetic field finite element analysis software can be used to analyze the package It includes static electric field, static magnetic field, time varying electric field, time varying magnetic field and eddy current meter is a powerful multi domain electromechanical system design and simulation analysis software. It is suitable for modeling, design, simulation analysis and optimization of electromechanical integration, such as electrical, electromagnetic, power electronics, control and so on. Maxwell can generate highly accurate equivalent circuit models that are available to software and other circuit analysis tools. In the joint simulation, Maxwell is used to analyze and simulate the electromagnetic field, and is mainly used for the analysis of the circuit. Co simulation using Maxwell and software in Qi standard wireless charging model of electromagnetic field and circuit, to verify and analysis of wireless charging model in Qi standard, and wireless charging experiment and optimization design for the future and lay a good foundation.

Principle of Electromagnetic Induction Wireless Charging. At present, most of the wireless charging using electromagnetic induction, including the current commercial wireless charging standards. Transmission of electromagnetic induction wireless charging energy through the coil and realize energy disaster, the basic principle is to set up 1 coils in sending and receiving end, the transmitter coil is connected with a high-frequency alternating wired power, and electromagnetic signal receiving coil receives the electromagnetic signal and change into the current, the current through rectifying and stabilizing after the circuit provides power to charge the battery. The equivalent schematic diagram of the wireless charging system is shown in figure 1. Suppose that $U$ is AC (voltage source) voltage. I, and $\mathrm{ZZ}$ respectively for transmitting and receiving coil current, $\mathrm{Z}$, 
and $\mathrm{ZZ}$ respectively represent the sending and receiving coil impedance; $\mathrm{R}$, and $\mathrm{RZ}$ respectively represent the equivalent resistance; $\mathrm{C}$ for transmitting coil series resonant capacitor; $\mathrm{CZ}$ receiving coil series resonant capacitor; $\mathrm{L}$ for transmitting coil inductance; LZ receiving coil inductance; $\mathrm{M}$ sending and receiving coil mutual inductance between $\mathrm{R}$ and load.

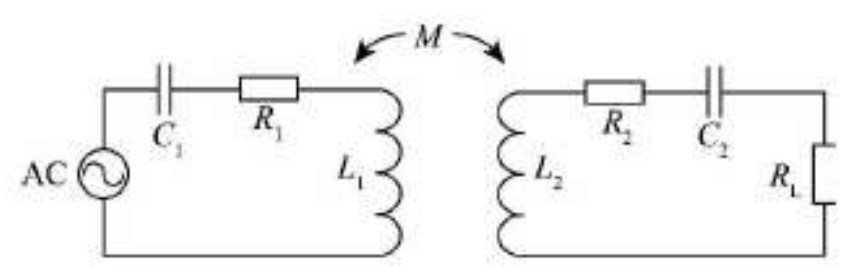

Figure 1. Finite electromagnetic induction wireless charging

Assuming that both the transmitter and receiver are in the optimal resonant state through the series connected resonant capacitor, the maximum transmission efficiency can be achieved at the transmitter and receiver. The resistance, capacitance and frequency parameters can be obtained by measuring, because the distance, offset and rotation of the coil changes, the coefficient of variation will change, and ultimately lead to changes in charging efficiency.

Maxwell Simulation Maxwell simulation software has a wizard type user interface, high-precision adaptive subdivision technology and powerful post-processing function. Maxwell 3 $\mathrm{D}$ is a high-performance 3D electromagnetic design software, Maxwell can analyze eddy current, displacement current and skin effect Proximity effect, such as motor, bus, transformer, coil and so on The impedance, force, torque, inductance, energy storage and other parameters can be self determined Dynamic calculation. At the same time, the magnetic field lines of the whole phase can also be given $\mathrm{BlH}$ distribution diagram, energy density, temperature distribution and other graphical results. Simplorer is an intuitive, multi domain, multi technology simulation soft The software allows engineers to simulate complex power electronics.

Maxwell Model Analysis The simulation steps of Maxwell 3 D model include choosing the type of solver, establishing 3D model, setting material attributes, setting boundary conditions, setting excitation, meshing, finite element calculation and result processing, etc.. Qi standard provides a variety of specifications of the transmission coil and receiving line Coils and detailed coil dimensions, materials and specified shielding materials are provided Attributes and so on. Selecting A1 coil in Qi standard by 3D simulation model By using the Maxwell finite element method, a more accurate knot can be obtained Maxwell 3 D model simulation solver type selection eddy current (eddy) mode, excitation source selection, current excitation and mesh generation The magnetic field intensity of the coil can be obtained by analyzing and processing the results of the matrix parameters Degree distribution, $\mathrm{R}$ (resistance), L (inductance), mutual inductance, coefficient of coincidence and so on.

In the design of wireless charging coil with ferrite shielding material has two main functions: 1) to enhance the strength of the magnetic field, magnetic flux leakage and provides 1 low impedance path for magnetic flux; absorption materials on the magnetic isolation; 2) increase the induction distance, improve the inductance and quality factor of the coil. Due to different permeability and frequency characteristics, ferrite materials have great influence on coil inductance and ferrite loss. the shielding coil ferrite material can use a variety of ferrite materials, different companies A, coil simulation choose ferrite material PE22 TDK, Faiu Rite, $M$ ateuial choose to receive the end coil shielding ferrite material 44 ferrite material. Compared with coil with ferrite shield and without ferrite shield, the coil capacitance with ferrite shield is obviously improved, and the coefficient of coincidence between coils is also improved. The receiving coil receives more flux, indicating that the higher between the 2 coil coupling degree, in the Qi standard system coupling coefficient range from 0.2-0.7, in the Qi standard by series resonant capacitor leakage inductance on the resonant compensation, can effectively solve the problem of low degree. The coupling between the factor 
and 2 coil distance, offset variation and its relative size, and also between the coil shape and the angle between the coil coupling coefficient changes will affect the charging efficiency.

Joint Simulation of Maxwell and Simplorer. Simplorer software provides a variety of ways to implement and Max-The interconnection of well software is shown in figure 4. In this paper, we choose the inclusion The method of state space equation, select Suhcir- under the menu Ciut->Maxwell component->Add state space In Simplorer software The icon of the Maxwell 3 D model appears in the front interface Providing external interface to connect with Simplorer circuit. Simplorer circuit simulation analysis using Maxwell software imitation The impedance matrix of the 3D model of the coil is generated, in which the impedance moment is obtained The array is preserved by the state space equation. When Maxwell and Simplorer are co simulation, M ax-Well is mainly responsible for the finite element analysis of the electromagnetic part of the coil The main parameters include coil inductance, mutual inductance and coincidence coefficient The finite element method is used to calculate the electrical signals generated by Simplorer Line is calculated and output to Simplorer for circuit simulation. The standard modeling language is convenient for modeling, digital and mixing Multi domain design model of signals. According to the hardware simulation circuit of the Qi standard definition of the practical design, joined the state machine network in the simulation circuit to generate a drive signal of the Mosfet tube, the driving signal to generate $50 \%$ duty cycle square wave signal is provided to the transmitting coil Mosfet half bridge tube, frequency from $110 \mathrm{kHz}$ to $205 \mathrm{kHz}$, the highest step adjustment; at the same time in order to see to realize the simulation initialization and simulation results, to add a power meter, voltage meter, initial state machine, set the value equation and network element to circuit simulation. As shown in Fig. 2, according to the transmitting coil and receiving The inductance of the coil and the resonant capacitor by proper selection of resonance circuit formula, the receiving coils are in series and parallel resonant capacitor, the series resonant capacitor is in order to enhance the efficiency and the parameters, the parallel resonant capacitor is needed in order to detect communication, parallel resonant capacitance due to the capacitance value is low on the overall efficiency of the system is less affected.

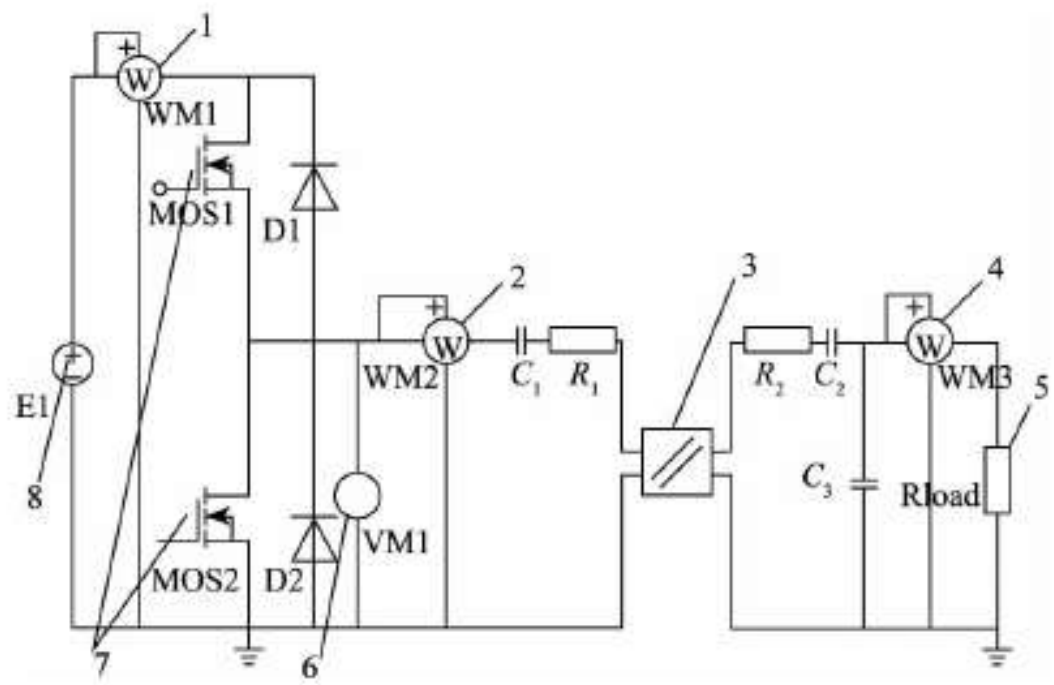

Figure 2. Finite joint simulation interface

In Simplorer, adding Maxwell 3 D coil engineering, Simplorer simulation will automatically call the coil impedance matrix generated by the Maxwell compiler and into the circuit operation, all results in the compiler after you can view the simulation in Simplorer. AC (frequency domain) Co simulation can get the gain and phase change of the load voltage RL varies with the frequency of the transmitting coil Bode, RL voltage gain at the frequency of $130 \mathrm{kHz}$ under the condition of maximum charging efficiency reached the highest value. The frequency of the highest efficiency 
can be obtained by using AC (frequency domain solution), and the TR (transient solution) mode joint simulation can obtain the various state parameters of the circuit at this frequency. The curve is charged at $130 \mathrm{kHz}$ frequency, and the output voltage of the voltage source and the output power of the half bridge and the load power of the M osfet tube are measured when the coil is in the gap of 3 $\mathrm{mm}$. The half bridge output power of Mosfet tube is slightly lower than the output power of voltage source due to the partial power loss caused by Mosfet internal resistance. The output power / load power of the Mosfet half bridge is compared by the power curve integral, and the efficiency is about $81 \%$.

\section{Result}

In the Simplorer simulation including AC (frequency domain solution) and TR (transient) simulation model, can solve the frequency change and change of circuit parameters including frequency domain model, and solve the transient mode by setting the simulation time and step time, can the simulation time and the circuit parameters. As shown in Fig. 5, the gain and phase variations of the load RL voltage varying with the frequency of the transmitting coil can be obtained by combining the AC (frequency domain solution) together with the true Bode The RL voltage gain reaches the maximum at the frequency of $130 \mathrm{kHz}$, and the charging efficiency reaches the highest value. The frequency of the highest efficiency can be obtained by using AC (frequency domain solution), and the TR (transient solution) mode joint simulation can obtain the various state parameters of the circuit at this frequency. The change curve of transmitting coil and receiving coil input current charging current at $130 \mathrm{kHz}$ frequency, coil in the gap is $3 \mathrm{~mm}$ under the condition of voltage source output power, after the change curve of $M$ osfet half bridge output power and load power. The half bridge output power of Mosfet tube is slightly lower than the output power of voltage source due to the partial power loss caused by Mosfet internal resistance. The output power / load power of the Mosfet half bridge is compared by the power curve integral, and the efficiency is about $81 \%$.

\section{Conclusion}

Through the joint simulation of Maxwell and Simplorer simulation software, to verify the effectiveness of the Qi standard wireless charging electromagnetic induction coil, the magnetic field strength of the Qi standard, inductance, resistance, coupling coefficient and ferrite loss was analyzed by Maxwell finite element analysis, finally to verify the Qi standard circuit co simulation using Maxwell and Simplorer software, the simulation results and actual results. Co simulation of Maxwell and Simplorer simulation software can achieve the prototype to validate the design, simulation and high precision, can join the United simulation bridge rectifier circuit, and according to the Qi standard and PID control method.

\section{References}

[1] HASANZADL typing technology in electrical power transmission systems Conversion an Management 2013, 65(10): 487-496.

[2] L1UZQ.YANGZ.Wire power supply system for artificial anal sphincter it experimental study China ,Journal of Scientific In-atrument2013，34(12): 2831-2838

[3] Z.B.Xu,The application and prospect of DNA analysis technology in forensic science, journal of law and medicine, 2000. 